\title{
ANÁLISIS FINANCIERO DE LAS OPERACIONES DE PRÉSTAMOS CON INDICIACIÓN EN LOS TIPO DE INTERES
}

\author{
Rosa María Cáceres Apolinario* \\ Departamento de Economía Financiera y Contabilidad \\ Universidad de Las Palmas de Gran Canaria \\ Juan García Boza \\ Departamento de Economía Financiera y Contabilidad \\ Universidad de Las Palmas de Gran Canaria \\ (Recibido 20 de febrero 2006, aceptado 27 de marzo 2006)
}

\begin{abstract}
Resumen
En la actualidad los préstamos a tipos de interés indiciados tienen una importancia creciente, por lo que han ido apareciendo en la práctica financiera distintas modalidades contractuales y diversos métodos de amortización que no han sido estudiados en profundidad, por lo que consideramos relevante su análisis desde la perspectiva financiera. Por ello en el presente trabajo realizamos un estudio de la problemática relativa a la determinación del tipo de interés en los préstamos indiciados así como un análisis financiero de las distintas metodologías de amortización aplicables a tales operaciones, distinguiendo fundamentalmente entre metodologías con términos predeterminados y con términos posdeterminados.
\end{abstract}

\section{Abstract}

The importance of the indexed loans has been increasing worldwide arising in the financial practice different contractual schemes and systems of amortization that have not been studied in depth. In this context is framed the present study in which we study the determination of the interest rate in the indexed loans, analysing from a financial perspective the different methodologies of amortization than can be apply to such operations, distinguishing between those ones with predetermined payments and post-determined payments.

Clasificación JEL: : G20, G21

Palabras clave: Tipos de interés indiciados, Préstamos, Metodologías de Amortización

* Universidad de Las Palmas de Gran Canaria. Facultad de Ciencias Económicas y Empresariales. Campus Universitario de Tafira, 35017 Las Palmas de Gran Canaria, España. Correo electrónico: rcaceres@defc.ulpgc.es 


\section{Introducción}

Debido a la continua variabilidad de los tipos de interés durante los últimos años, los préstamos a tipos de interés variables han tomado importancia creciente, y de forma especial los vinculados a garantías hipotecarias, así como aquellos otros en los que el tipo de interés es función de algún índice previamente acordado (operaciones con tipos de interés referenciados o indiciados ${ }^{1}$ ). Dada la relevancia de tales préstamos, han ido apareciendo en la práctica financiera distintas modalidades contractuales y diversos métodos de amortización que, no obstante, no han sido estudiados de forma científica, por lo que consideramos de interés su análisis desde la perspectiva financiera.

Las características financieras de las operaciones con tipos vinculados a variaciones de índices, así como los usos del mercado, ponen de manifiesto la existencia de una variada problemática que entendemos no ha sido suficientemente analizada desde la óptica cuantitativa, por lo que parece preciso adaptar los métodos clásicos de amortización de préstamos, estudiados con profusión en la Matemática Financiera, a tales características. Por ello vamos a realizar en nuestro trabajo un análisis financiero de las distintas metodologías de amortización aplicables a tales operaciones. En este sentido, analizaremos en primer lugar la problemática vinculada a la determinación de los tipos de interés indiciados, y posteriormente, nos detendremos en el estudio de las metodologías de cuantificación de las distintas variables financieras que conforman el plan de amortización de las operaciones mencionadas.

El análisis citado lo realizaremos bajo la hipótesis de que el préstamo sea amortizado con contraprestación múltiple, o sea, entregando el deudor un conjunto de términos amortizativos, clasificando los distintos métodos en función de las cuantías de los términos amortizativos o cuotas totales a pagar periódicamente por el prestatario. En este sentido, podemos distinguir entre metodologías de amortización con términos predeterminados, en las que las cuantías de los mismos son acordadas en el origen de la operación, y metodologías de amortización con términos posdeterminados, en las que las cuantías de los mismos son desconocidas al inicio de la operación. En cada una de las metodologías citadas, profundizaremos en el estudio de distintas modalidades en función de las características que se pueden pactar entre los sujetos de tales operaciones.

\section{Problemática de la determinación de los tipos de interés indicia- dos}

Tal y como hemos señalado, nuestro objetivo fundamental en este trabajo está relacionado con toda la problemática cuantitativa derivada de las operaciones de amortización de los préstamos pactados con tipos de interés indiciados. Por ello, y a fin de enmarcar claramente dichas operaciones, nos parece clasificar las operaciones de amortización, distinguiendo distintas modalidades en función de los tipos de interés que permiten cuantificar el precio financiero de la correspondiente operación.

1 La palabra indiciación es sinónimo de indexación (indexation en inglés) y significa la vinculación de una operación financiera, en cuanto al tipo de interés aplicable, a una variable económica, normalmente al tipo de interés que se establezca en un determinado mercado como el Euribor o el Libor. 
Tomando el tipo de interés como la variable financiera más relevante a efectos de una conceptualización y clasificación de las operaciones de préstamo, distinguimos entre aquellas operaciones pactadas con tipos predeterminados y las acordadas con tipos posdeterminados.

Cuando se pacta la modalidad de tipos predeterminados, los mismos son conocidos desde el inicio de la respectiva operación, pudiendo ser el tipo constante a lo largo de toda la vida de la misma; o por el contrario, ser variable, en cuyo caso se establecen distintos tipos de interés para aplicar en los diversos períodos temporales hasta la amortización del préstamo.

En la modalidad de tipos posdeterminados, en el origen de la respectiva operación no son conocidos todos los tipos de interés a aplicar durante la vida de la misma, por lo que al inicio de cada período temporal ha de fijarse el tipo de interés correspondiente al mismo. En este caso, las partes contratantes de las operaciones pueden optar por las siguientes alternativas:

a) Sin indiciación. En tal supuesto, al inicio de la operación, no se vincula la determinación de los tipos de interés a variable alguna, distinguiéndose dos clases en función del sujeto o sujetos que tengan la potestad de establecer los mencionados tipos: los préstamos a tipo revisable y los préstamos a tipo renegociable. Cuando los tipos sean revisables, es el sujeto prestamista el que determina el tipo de interés a aplicar en el período correspondiente, siendo uno de los criterios de decisión la evolución de sus costes de refinanciación. En el supuesto de tipos renegociables, ambas partes contratantes establecen de común acuerdo el tipo de interés a aplicar.

b) Con indiciación. En este caso, también los tipos de interés se fijan al inicio del correspondiente intervalo temporal de su vigencia pero vinculándose su cuantía a una variable de referencia, previamente pactada en las condiciones de la respectiva operación. Ello da origen a los préstamos con tipos de interés referenciados o indiciados en las cuotas de interés, siendo ésta la modalidad de mayor utilización en las operaciones concertadas por entidades financieras.

En los préstamos con tipos referenciados, a los efectos de la determinación de los sucesivos tipos de interés, se establece una frecuencia o plazo de revisión, de amplitud $t$ períodos unitarios, al principio del cual se calcula el tipo de interés a aplicar hasta la siguiente revisión. Este plazo de revisión ha de ser necesariamente establecido en el contrato que sustenta la operación de préstamo, y puede ser de igual o mayor amplitud temporal que la correspondiente frecuencia de los pagos del prestatario o periodicidad de amortización.

El tipo de interés nominal vigente durante $t$ períodos unitarios y hasta la próxima revisión del mismo, se determina como suma del valor que tenga el índice de referencia con el margen o diferencial, ${ }^{2}$ verificándose:

$$
j=j_{r} \pm m
$$

siendo: $j$ :tipo de interés nominal por unidad de tiempo, $j_{r}$ : índice de referencia, $m$ : margen o diferencial

2 En algunas operaciones, los prestamistas aplican a esta suma un redondeo a fracciones de $\frac{1}{4} \frac{1}{8}$ ó $\frac{1}{16}$. 
Los distintos índices de referencia son normalmente establecidos por las autoridades financieras competentes, adhiriéndose a ellos los sujetos contratantes de cada operación y pactando el que consideren más adecuado a las características de la misma.

El margen o diferencial es una cuantía que modifica al índice de referencia en la fecha de revisión del tipo de interés ${ }^{3}$. El mismo puede ser fijo, pactado al inicio de la vida del préstamo y constante durante toda ella; o bien variable, modificándose en alguno o en todos los plazos de revisión.

La metodología descrita para calcular el tipo de interés nominal aplicable se suele ver complementada habitualmente con límites máximos y/o mínimos en el tipo de interés, previamente pactados al inicio de la operación. Su justificación se encuentra en las posibles variaciones extraordinarias, tanto al alza como a la baja, en el índice de referencia utilizado.

Si el proceso descrito para obtener los distintos tipos de interés se aplica durante toda la vida del préstamo, estamos ante una indiciación total de los mismos. Cuando la metodología de indiciación sólo se utiliza para un cierto intervalo temporal, nos encontramos con una indiciación parcial.

En la hipótesis de indiciación parcial combinamos tipos de interés referenciados con tipos constantes, de manera que en la operación existe al menos un intervalo temporal en el que el tipo de interés es constante y al menos otro en el que el tipo de interés es referenciado o indiciado. Dentro de las múltiples modalidades que se pueden dar, destacamos las tres siguientes:

Una primera modalidad es aquella en la que durante los primeros $s$ períodos ${ }^{4}$ el tipo de interés a aplicar es constante y previamente establecido en el origen de la operación. En los restantes períodos, el tipo de interés se obtiene según la metodología descrita anteriormente. En consecuencia, existe un intervalo temporal en el que los tipos son predeterminados, o intervalo con tipo fijo, y a continuación pasan a ser posdeterminados.

La segunda modalidad también implica el acuerdo sobre un determinado tipo vigente durante un cierto plazo, por lo cual durante los s períodos iniciales el tipo a aplicar es constante y predeterminado. Al finalizar dichos períodos, el deudor puede optar entre continuar con la operación a tipo fijo, o bien cambiar a una operación con tipos referenciados. Por tanto, para los períodos siguientes a los $s$ primeros, tenemos las dos opciones siguientes: a) Tipo constante acordado entre ambas partes contratantes y aplicable durante un número de períodos $t^{\prime}$ igual o superior al del plazo de revisión $t: t^{\prime} \geq t ;$ o b) Tipo indiciado vigente durante $t$ períodos. Transcurridos los períodos correspondientes, $t^{\prime}$ o $t$ , nuevamente el prestatario puede optar por cualquiera de las dos alternativas citadas, tipo constante o tipo referenciado, que serán aplicables durante los siguientes $t^{\prime}$ o $t$ períodos. Este proceso se repite hasta la finalización de la

3 Normalmente, el margen o diferencial se suma al índice de referencia para obtener el tanto de interés. No obstante, también se pueden pactar operaciones en las que el margen estipulado tenga un efecto multiplicativo, obteniéndose el tanto nominal según la siguiente expresión: $j=j_{r} m$.

4 Es habitual en la práctica bancaria que se verifique $s=t$. 
operación. 5

Una tercera posibilidad es aquella en la que durante los $s$ períodos iniciales, previamente pactados, se aplican los tipos de interés indiciados que correspondan al índice de referencia y margen establecidos. En los períodos siguientes hasta la finalización de la operación, el tipo de interés es constante e igual al tipo medio de coste de la operación ya vencida, que denominamos tipo medio de coste retrospectivo. Dicho coste medio se obtiene como el tipo de interés constante con el que se verifica la ecuación de equivalencia financiera entre los términos amortizativos vencidos, el saldo pendiente de amortizar y la cuantía objeto del préstamo:

$$
C\left(1+\bar{i}_{s}\right)^{s}=\sum_{r=1}^{s} a_{r}\left(1+\bar{i}_{s}\right)^{s-r}+C_{s} \Rightarrow \bar{i}_{s}
$$

siendo: $C$ : importe del préstamo, $C_{s}$ : saldo o capital vivo al final del período $s$, $a_{r}$ : término amortizativo desembolsado al final del período $r \operatorname{con} r=1,2,3 \cdots, s$ y $\bar{i}_{s}$ : tipo medio de coste retrospectivo.

En esta última modalidad de préstamos con indiciación parcial, al no conocerse al inicio de la operación ninguno de los tipos de interés que se van a aplicar hasta finalizar los $s$ períodos, el tipo constante medio que resulte de los mismos tampoco es conocido, por lo que nos encontramos en presencia de una modalidad de préstamo con tipos posdeterminados

De acuerdo con la clasificación señalada, nos vamos a centrar en los siguientes epígrafes de este trabajo en el análisis de la problemática relativa a los préstamos en los que existe indiciación en los tipos de interés, ya sea la misma total o parcial. Para ello vamos a distinguir entre metodologías de amortización con términos predeterminados y metodologías de amortización con términos posdeterminados.

\section{Metodologías de amortización con términos predeterminados}

La hipótesis básica de estas metodologías implica que desde el inicio de la operación son conocidas las cuantías de los términos amortizativos que debe desembolsar el deudor, ya sean constantes o variables, las cuales son acordadas entre las partes contratantes de la operación. En consecuencia, al estar los intereses vinculados tanto al saldo pendiente de amortizar como al tipo de interés referenciado correspondiente, es evidente que las distintas cuotas de amortización que permiten la devolución de la cuantía objeto del préstamo, también son desconocidas, por lo que la duración de la operación se desconoce a priori, ya que vendrá determinada por la evolución del tipo de interés. Si bien el plazo $n$ de la operación es indeterminado, se puede establecer una limitación

5 También es posible pactar que desde el origen de la operación, y sin que transcurran $s$ períodos, se pueda optar por cualquiera de las dos alternativas citadas. En tal supuesto, pudiera suceder que el deudor no eligiese en ningún caso la modalidad de tipo constante, encontrándonos, pues, al finalizar la vida de la operación, que la misma ha sido con indiciación total. $Y$, en cambio, si ha elegido siempre un tipo constante por intervalos de amplitud $t^{\prime}$, estaríamos en presencia de una operación con tipos posdeterminados renegociables y consecuentemente, sin indiciación. 
temporal, de forma que el número de períodos no exceda de un límite máximo $n^{*}: n \leq n^{*}$, dando lugar a dos modalidades: a) préstamos con duración máxima predeterminada; y b) préstamos sin duración máxima.

$\mathrm{Al}$ ser en estos métodos conocidos los términos amortizativos, y con el objetivo de establecer una duración inicial de referencia, vamos a suponer que el tipo de interés aplicable hasta la primera revisión del mismo, permanece constante durante toda la vida del préstamo. En tal hipótesis, independientemento de que los términos amortizativos sean constantes o variables, es posible determinar dicha duración inicial de referencia $h$, deduciéndola de la ecuación de equivalencia financiera entre prestación y contraprestación al tipo de interés señalado $i_{1}$ :

Si $a_{s}=a, \forall s \mid s=1,2,3, \cdots h$, se verifica: $C=a a_{\bar{h} \mid i_{1}} \Rightarrow h$, siendo $a_{\bar{h} \mid i_{1}}=\frac{1-\left(1+i_{1}\right)^{-h}}{i_{1}}$. Si $a_{s}$ es variable, $\forall_{s} \mid s=1,2,3 \cdots h$, se verifica: $C=$ $\sum_{s=1}^{h} a_{s}\left(1+i_{1}\right)^{-s}$. Como las cuantías $a_{s}$ se modifican según una determinada ley de variación, ${ }^{6}$ será posible hallar el valor de $h$.

La hipótesis introducida permite también obtener las cuotas de interés y de amortización iniciales de referencia en cada uno de los $h$ períodos de duración inicial, debiendo verificarse, cuando se haya pactado una duración máxima que $h \leq n^{*}$.

En la hipótesis de que la evolución temporal del tipo de interés referenciado sea creciente, $i_{s}<i_{s+1} \mid s=1,2,3, \cdots, n-1$, o bien que el mismo en todos los períodos tome un valor superior al del primer período, ${ }^{7} i_{1}<i_{s} \mid s=$ $1,2, \cdots, n$, las cuotas de interés reales superarán en los respectivos períodos, a las correspondientes de referencia. Por consiguiente, las cuotas de amortización serán inferiores a las de referencia, con lo que la duración real superará a la inicial de referencia $h<n$. Si además se ha pactado un plazo máximo de duración del préstamo, debe verificarse: $h<n \leq n^{*}$.

Si la evolución temporal del tipo de interés referenciado es decreciente o bien el mismo en todos los períodos toma un valor inferior al del primero, las cuotas de interés reales resultarán inferiores en los respectivos períodos, a las correspondientes de referencia. En consecuencia, las cuotas de amortización serán superiores a las de referencia, siendo la duración real inferior a la inicial de referencia

$$
\left.\begin{array}{c}
i_{s}>i_{s+1} \mid s=1,2, \cdots, n-1 \\
i_{1}>i_{s} \mid s=2, \cdots, n
\end{array}\right\} \Rightarrow n<h,
$$

Verificándose que $n<h \leq n^{*}$ si se ha pactado un plazo máximo de duración del préstamo.

Tal y como hemos señalado, la evolución de los tipos de interés afecta a la duración real de la operación. Por ello, una vez abonado cada término

6 En este caso debe existir un criterio de variabilidad de los términos amortizativos, ya que en caso contrario, al desconocerse la duración de la operación, no sería posible establecer las cuantías correspondientes.

7 Conviene señalar que dicho tipo de interés es el utilizado para determinar la duración inicial de referencia. 
amortizativo, es posible determinar el número teórico de períodos pendientes hasta la total amortización del préstamo, considerando que el tipo de interés va a permanecer constante. Así, situados al inicio del período $s+1$, siendo $C_{s}$ el capital pendiente de amortizar en dicho momento, el número de períodos pendientes $h_{s}$ se deduce de la siguiente ecuación: $C_{s}=\sum_{t=1}^{h_{s}} a_{s+t}\left(1+i_{s+1}\right)^{-t}$. Una alternativa a la utilización del tanto $i_{s+1}$ para obtener el número de períodos pendientes, es tomar como tipo de actualización, el tanto medio de coste retrospectivo correspondiente ${ }^{8} \bar{i}_{s}$.

De acuerdo con lo expuesto, debido a la evolución de la operación, puede suceder que la duración real vaya a superar al límite máximo si éste ha sido establecido: $n>n^{*}$, por lo que es en el período correspondiente a dicho límite máximo, en el que la operación debe quedar íntegramente amortizada. Para ello será preciso modificar adecuadamente la cuantía del último término amortizativo a fin de que permita una cuota de amortización que posibilite liquidar la operación. En tal caso, dicho último término será de cuantía superior a la inicialmente prevista. En cambio, cuando no se haya establecido plazo máximo, el último término tendrá generalmente una cuantía inferior a la inicialmente prevista.

Tal y como hemos señalado, una vez establecidas las cuantías de los términos amortizativos, podemos deducir una duración inicial de referencia. No obstante, también se puede pactar dicha duración inicial y en función de la misma obtener las cuantías que debe abonar el deudor, pudiéndose establecer, además, un límite máximo para la duración real. En consecuencia, siempre existirá una duración inicial de referencia: bien la deducida de la equivalencia con los términos amortizativos predeterminados, o bien la pactada, en función de la cual se obtienen los términos amortizativos. En cualquier caso, podemos distinguir dos modalidades en función de que las cuantías de los términos predeterminados sean constantes o variables, y dentro de cada una de ellas, hemos de tener en cuenta si tales cuantías han sido establecidas previamente, o bien se obtienen en función de la duración inicial pactada como referencia. En consecuencia, las cuantías de los términos amortizativos se pueden determinar según las modalidades metodológicas que se desarrollan a continuación.

a) Modalidad 1: Términos amortizativos previamente establecidos y de cuantía constante: $a_{s}=a, \forall_{s} \mid s=1,2,3, \cdots, n$. Esta cuantía se puede obtener de las siguientes formas:

a.1) Como el importe máximo definido por la capacidad de pago periódica $a^{*}$ del deudor para hacer frente a tales términos: ${ }^{9} a=a^{*}$.

8 Recordemos que dicho tanto medio viene dado como la raíz de la ecuación de equivalencia financiera entre los capitales recibidos y entregados por el deudor, adicionando a éstos el saldo pendiente de amortizar.

9 Esta cuantía máxima es establecida por las entidades prestamistas siguiendo determinados criterios económico-financieros aplicados a los estados contables del deudor. Uno de los parámetros más utilizados es el Payment to Income, que es el cociente entre el importe del término amortizativo y la renta disponible, de forma que dicha ratio no exceda de un determinado valor que de manera general oscila en torno al $30 \%$. No obstante, al tratarse de operaciones a largo plazo, con la dificultad que ello implica para determinar cuantías máximas futuras, no siempre se establece tal límite máximo. 
a.2) Utilizando un método proporcional, mediante el cual la cuantía del término amortizativo constante se obtiene en proporción directa a la primera cuota de interés, con coeficiente de proporcionalidad $p>1: a=C i_{1} p \Rightarrow A_{1}=C i_{1}(p-1)$. a.3) Para aquelios préstamos en los que se haya estipulado una duración máxima, la cuantía constante del término amortizativo se puede obtener de la ecuación de equivalencia financiera, al tipo de interés del primer período, y tomando como duración el límite máximo $n^{*}$ acordado: $C=a a_{\bar{n}^{*} I_{1}} \Rightarrow a$. No obstante, ante una evolución creciente de los tipos de interés, sus efectos sobre las cuotas se van a ir acumulando y pudiera suceder que en el último período, en el que es preciso ajustar el término amortizativo para que el préstamo quede cancelado, la cuantía del mencionado término fuese excesivamente superior a las precedentes, lo que podría hacer que.el deudor tuviese dificultades para hacer frente a su pago. Por ello, y con el fin de evitar parcialmente tal situación, se puede establecer un número de períodos de seguridad $d$ de tal forma que el término amortizativo se calculase para un tiempo $n^{*}-d: C=a a \overline{n^{*}-d_{l}} 1_{1} \rightarrow a$. La cuantía así obtenida, obviamente supera a la determinada para una duración $n^{*}$. El número de períodos de seguridad se puede establecer en función de las expectativas en cuanto a la evolución futura de los tipos de interés que el prestamista tenga en el momento de conceder el préstamo.

a.4) Otra alternativa para la hipótesis en la que se haya establecido una duración máxima, es adicionar a la primera cuota de interés una cuota teórica de amortización calculada como cociente entre la cuantía del préstamo y la duración máxima pactada:

$$
a=i_{1} C+\frac{C}{n^{*}}=\frac{C\left(1+n^{*} i_{1}\right)}{n^{*}}
$$

b) Modalidad 2: Términos amortizativos de cuantía constante determinados en función de la duración inicial pactada $h$. Esta cuantía se obticne considerando que el tipo de interés inicial permanece constante:C $=a a_{\bar{h} \mid 1_{1}} \Rightarrow a$.

c) Modalidad 3: Términos amortizativos previamente establecidos y de cuantía variable $a_{s}$, pudiendo existir en algunos casos una cota superior tal que $a_{s} \leq$ $K$. La ley de variabilidad para tales términos puede ser cualquiera, aunque lo habitual es la variación en progresión aritmética o geométrica de razón, $d$ ó $q$, respectivamente, establecida por las partes: $a_{s}=a_{s-1}+d ; a_{s}=a_{s-1} q ; \forall s \mid s=$ $2,3, \cdots, n$.

Conocida la ley de variación de los términos, nuestro interés se centra en determinar el primero de los mismos. Con tal objetivo se puede utilizar cualquiera de los métodos descritos en la modalidad 1. Ahora bien, en el supuesto de que los términos amortizativos sean crecientes, no es posible aplicar la modalidad a.1) anteriormente descrita, ya que carece de sentido que el primer término coincida con la cuantía máxima que el deudor pueda satisfacer.

d) Modalidad 4: Términos amortizativos de cuantía variable determinada en función de la duración inicial pactada $h$, estableciéndose también, si así lo acuerdan las partes, una cuantía máxima para tales términos. La ley de variación de los términos puede ser cualquiera, aunque habitualmente se pacta la variación en progresión aritmética o geométrica, y considerando collstante el tipo de interés inicial, es posible determinar a través de la ecuación de equivalencia financiera, el primer término amortizativo, y a partir del mismo, los siguientes. 
Así, cuando la variación sea en progresión aritmética, de la conocida expresión general se deduce la cuantía del primer término:

$$
C=\left[a_{1}+d h+\frac{d}{i_{1}}\right] a_{\bar{h}_{\mid i_{1}}}-\frac{d h}{i_{1}}
$$

Y cuando la variación sea en progresión geométrica, de la siguiente ecuación se obtiene el primer término:

$$
C=\frac{a_{1}\left[1-q^{h}\left(1+i_{1}\right)^{-h}\right]}{1+i_{1}-q}
$$

En cualquiera de las modalidades citadas para la hipótesis en que los términos amortizativos sean de cuantía predeterminada, una vez obtenidos los mismos, al ir conociéndose el tipo de interés de cada período, se determinan las sucesivas cuotas de amortización, lo que nos llevará a establecer el período concreto n en el que el capital queda totalmente amortizado: $n \mid \sum_{s=1}^{n} A_{s}=C$.

\section{Metodologías de amortización con términos posdeterminados}

En estas metodologías no se establece a priori la cuantía de los términos amortizativos, pudiéndose, sin embargo, en determinados supuestos conocerse la duración de la operación, o bien en otros, ser desconocida. Ello da lugar a dos métodos: a) plan de amortización predeterminado, en el que se conoce desde el inicio de la operación tanto la duración de la misma como las cuantías de las cuotas de amortización; b) plan de amortización posdeterminado, en el cual son desconocidas las cuotas de amortización, pudiéndose fijar la duración de la operación o bien que la misma sea indeterminada, en cuyo caso se suele pactar un límite máximo para ella. A continuación analizamos cada una de las distintas opciones.

\subsection{Método con plan de amortización predeterminado}

En este método se conoce en el origen de la operación la cuantía correspondiente a cada una de las cuotas de amortización por lo que la duración es conocida, verificándose: $A_{s} \mid \sum_{s=1}^{n} A_{s}=C_{0}$. En consecuencia, la problemática que se presenta en este método es la determinación de las cuantías de las cuotas de capital o de amortización, ya que las cuotas de interés se determinarán en función del tipo de interés referenciado correspondiente. Al adicionar cada cuota de capital con la correspondiente cuota de interés que se obtenga, hace posible obtener la cuantía de cada término amortizativo. Ello da lugar a la existencia de dos modalidades: una en la que dichas cuotas de amortización son constantes; y otra, con cuotas variables.

a) Modalidad 1: Cuotas de amortización de cuantía constante. El importe de cada una de las cuotas de capital se puede determinar por el método lineal o italiano, o bien utilizando el método proporcional. En la hipótesis de utilizar el método italiano, se verifica: $A_{s}=A=\frac{C}{n}, \forall s \mid s=1,2,3, \cdots n$. En cambio, en el método proporcional, la cuantía constante de cada cuota de capital es proporcional a la de la primera cuota de interés, quedando determinada en el origen de la operación la duración de la misma, ya que en ese momento se 
conoce el tipo de interés a aplicar al primer período. Siendo $p$ el coeficiente de proporcionalidad, ${ }^{10}$ se verifica: $A_{s}=A=C i_{1} p, \forall s \mid s=1,2,3, \cdots, n ; n=\frac{1}{i_{1} p}$.

b) Modalidad 2: Cuotas de amortización de cuantía variable. El importe de estas cuotas puede ser fijado arbitrariamente de tal forma que permitan la amortización del capital, o bien seguir un determinado criterio de variación, el cual puede ser un criterio aritmético o lineal, o uno geométrico. En cualquiera de estos dos últimos casos, una vez determinada la primera cuota, las restantes se deducen de ésta por aplicación del criterio analítico correspondiente.

En el criterio aritmético o lineal, las cuotas de amortización varían en progresión aritmética de razón dada $d$, siendo $A_{s}=A_{s-1}+d$, verificándose: ${ }^{11} A_{1}=\frac{C}{n}-$ $\frac{d(n-1)}{2}$ y $A_{n}=\frac{C}{n}+\frac{d(n-1)}{2}$. La razón pactada puede ser positiva o negativa. En cualquier caso, debemos establecer las condiciones que la misma debe cumplir a fin de que todas las cuotas de amortización sean positivas. Así, para $d>0$, si la primera cuota de amortización es positiva, las demás también lo serán, por lo que es necesario que la razón cumpla la siguiente limitación: ${ }^{12} 0<d<\frac{2 c}{n(n-1)}$. En la hipótesis de que sea $d<0$, si la última cuota de amortización es positiva, también lo serán las anteriores, debiéndose verificar: ${ }^{13} \frac{-2 C}{n(n-1)}<d<0$. En cl criterio lineal descrito, la razón $d$ puede ser libremente pactada por las partes, o bien establecerla en función de la primera cuota de interés o de la primera cuota de amortización, como un tanto unitario de cualquiera de ellas. En cualquier caso, siempre se debe cumplir la correspondiente desigualdad anterior.

Si la razón se pacta en función de la cuota de interés correspondiente al período primero, $d=I_{1} p=C i_{1} p$, y según sea el signo de $p$, será el correspondiente a $d$. En general, el importe de la primera y de la última cuota de amortización es, ${ }^{14}$ respectivamente: $A_{1}=\frac{C\left[2-i_{1} p(n-1) n\right]}{2 n}$ y $A_{n}=\frac{C\left[2+i_{1} p(n-1) n\right]}{2 n}$. Cuando sea $p>0$, será $d>0$, por lo que la limitación correspondiente se convierte en: $0<p<\frac{2}{i_{1} n(n-1)}$. En el supuesto de $p<0$, será $d<0$, por lo que la restricción correspondiente es: $\frac{-2}{i_{1} n(n-1)}<p<0$.

Si la razón se pacta en función de la primera cuota de amortización, $d=A_{1} p$, verificándose en general: $A_{1}=\frac{2 C}{n[2+p(n-1)]}$ y $A_{n}=\frac{2 C[1+p(n-1)}{n[2+p(n-1)]}$. Cuando sea $p>0 \Rightarrow d>0$, de la ecuación precedente de la primera cuota de amortización, se deduce que la misma toma siempre valores positivos con independencia del valor asignado a $p$, no existiendo, pues, restricción alguna cuando sea $p$ positivo. Para $p<0 \Rightarrow d<0$, si la última cuota de amortización es positiva, las anteriores también lo serán, por lo que es necesario que se verifique: ${ }^{15} 1+p(n-1)>0 \Rightarrow \frac{-1}{n-1}<p<0$.

10 Este coeficiente ha de ser pactado entre las partes, siendo la única restricción aplicable al mismo, que sea positivo.

11 Estas expresiones se deducen teniendo en cuenta que $C=\sum_{s=1}^{n} A_{s}=\frac{\left(A_{1}+A_{n}\right) n}{2}$, siendo $A_{n}=A_{1}+d(n-1)$.

12 Deducida de $A_{1}=\frac{C}{n}-\frac{d(n-1)}{2}>0$.

13 La inecuación se deduce de: $A_{n}=\frac{C}{n}+\frac{d(n-1)}{2}>0$.

14 Ecuaciones obtenidas al sustituir $d \stackrel{n}{=} C \times i_{1} \times p$ en las ecuaciones de $A_{1}$ y $A_{n}$.

15 Teniendo en cuenta que $A_{n}=A_{1}[1+p(n-1)]$, como siempre ha de ser $A_{1}>0$, 
En el caso de utilizar el criterio geométrico, según el cual las cuotas han de variar en progresión geométrica de razón dada $q$, sabemos que $A_{s}=A_{s-1} q$, verificándose $^{16} A_{1}=\frac{C(q-1)}{q^{n}-1}$ y $A_{n}=\frac{C\left(q^{n}-q^{n-1}\right)}{q^{n}-1}$. La razón $q>0$ puede ser libremente pactada por las partes, siendo un caso particular aquel en el que $q=\left(1+i_{1}\right)$. Al sustituir en la expresión precedente, se llega a: $A_{1}=\frac{C i_{1}}{\left(1+i_{1}\right)^{n}-1}=$ $\frac{C}{s_{\tilde{n} ; i_{1}}}$. El plan de amortización obtenido de la forma descrita coincide con el de un préstamo amortizable en los $n$ períodos pactados, por el método francés al tipo constante del primer período.

\subsection{Método con plan de amortización posdeterminado}

Se caracteriza por desconocer al inicio de la operación las cuantías de las cuotas de capital y las de los términos amortizativos. Este método presenta una amplia problemática, ya que en el origen de la operación sólo es conocida la cuantía del préstamo, por lo que se debe pactar la metodología de cálculo de los términos amortizativos o bien la de las cuotas de amortización. Además, en función de que la duración sea establecida o no, se originan dos modalidades: con duración determinada y con duración indeterminada. A continuación se desarrollan las mismas.

\subsubsection{Método con plan de amortización posdeterminado y duración determinada.}

En este método se establece al inicio de la operación su duración así como la metodología que posibilitará el cálculo de cada cuota de amortización o de cada término amortizativo. Una alternativa a lo expuesto es la consistente en que una vez definida la duración, al inicio de cada período se pacta la cuantía correspondiente al término amortizativo del mismo o la de la cuota de amortización, de tal forma que la última cuota de capital posibilite la amortización íntegra del préstamo.

Si lo pactado entre las partes contratantes es la metodología de cálculo de las cuotas de amortización, una de ellas es el método proporcional en el cual tales cuotas se obtienen aplicando un porcentaje convenido a la respectiva cuota de interés: $A_{s}=C_{s-1} \dot{i}_{s} p$, siendo $p$ el coeficiente de proporcionalidad acordado. ${ }^{17}$ No obstante, para el cálculo de la cuota de amortización del último período no es posible aplicar este criterio, ya que tal cuota ha de ser coincidente con el saldo vivo al principio de dicho período, para así amortizar íntegramente la cuantía del préstamo en la duración establecida.

Cuando se haya pactado la metodología que posibilita la determinación de los términos amortizativos, la misma nos permitirá obtener las cuantías de tales términos, deduciéndose a continuación las correspondientes cuotas de amortización o plan de amortización. En este sentido, podemos utilizar, adaptándolos

se deduce la expresión que se indica. Además, cabe preguntarse si el denominador de la expresión de $A_{n}$ puede ser negativo. No es posible que lo sea, pues si lo fuese sería $A_{1}<0$, lo cual es absurdo.

16 Teniendo en cuenta la ecuación correspondiente a la suma de todos los términos de una progresión geométrica, hemos deducido las expresiones citadas.

17 Si se eligiese cuotas constantes, estaríamos en presencia de la modalidad ya analizada que corresponde al método con plan de amortización predeterminado. 
a las características propias de los préstamos indiciados, los métodos clásicos de amortización de préstamos (método francés, método de térninos amortizativos variables en progresión aritmética; y el de términos amortizativos variables en progresión geométrica ${ }^{18}$ ), dando origen a los métodos clásicos generalizados.

La metodología señalada supone para determinar dichos términos lo siguiente: al principio de cada plazo de revisión y con el tipo de interés del mismo considerándolo constante para el resto de la vida de la operación, se plantea la ecuación de equivalencia financiera entre el saldo vivo en dicho momento y las cuantías de los términos amortizativos, de acuerdo con las hipótesis establecidas para dichas cuantías en función del método tradicional elegido. Por tanto, a efectos de calcular los $\mathrm{n}$ términos amortizativos es preciso plantear de la forma descrita tantas ecuaciones de equivalencia financiera como número de revisiones del tipo de interés se deba efectuar.

Situados al principio del período $(s+1)$, momento en el cual se efectúa la revisión del tipo de interés, y considerando dicho tipo constante hasta el final de la operación, podemos representar gráficamente la parte de la operación pendiente y obtener la ecuación de equivalencia financiera correspondiente, y de esta forma determinar las cuantías de los términos amortizativos:

$$
C_{s}=\sum_{h=1}^{n-s} a_{s+\hbar}^{(s)}\left(1+i_{s+1}\right)^{-h}
$$

siendo: $C_{s}$ : saldo vivo o capital pendiente de amortizar al principio del período $(s+1), i_{s+1}$ : tipo de interés del período $(s+1), a_{s+h}^{(s)}$ : término amortizativo correspondiente al período $(s+h)$ calculado en función del saldo en $s$.

Es preciso destacar que de acuerdo con la metodología utilizada, el tipo de interés $i_{s+1}$ es el que se supone aplicable en todos los períodos futuros a los únicos efectos de determinar los términos amortizativos correspondientes a los $t$ períodos siguientes hasta la próxima revisión del tipo de interés. $\mathrm{Al}$ considerar que el tipo de interés es revisado cada $t$ períodos, la expresión [7] del saldo la podemos descomponer en dos sumandos, de forma que el primero indique el valor actual de los $t$ términos amortizativos que se han de abonar hasta la siguiente revisión del tipo de interés, y el segundo, el valor actual de los restantes términos amortizativos calculados en función del saldo en $s$ :

$$
C_{s}=\sum_{p=1}^{t} a_{s+p}^{(s)}\left(1+i_{s+1}\right)^{-p}+\sum_{q=i+1}^{n-s} a_{s+q}^{(s)}\left(1+i_{s+1}\right)^{-q}
$$

Las cuantías $a_{s+p}^{(s)}$ con $p=1,2, \cdots t$, son las que realmente debe entregar el prestatario en cada uno de los $t$ períodos hasta la próxima revisión del tipo de interés, por lo que le damos el calificativo de términos amortizativos efectivos. Los siguientes términos, que son utilizados únicamente para calcular los

18 Pudiera pensarse en la posibilidad de incluir también el sistema italiano. Sin embargo, ello no es posible, pues en el mismo, las cuotas de amortización son constantes y conocidas desde el inicio de la operación, por lo que nos encontramos ante una modalidad del método con plan de amortización predeterminado, ya estudiado. 
$t$ primeros citados, ya que no se corresponden con las cuantías que debe abonar el deudor en los siguientes períodos, los denominamos términos amortizativos teóricos. En definitiva, los términos efectivos correspondientes a los $t$ períodos hasta la siguiente revisión, son los que se determinan teniendo en cuenta el tipo de interés, ya conocido, a aplicar en dichos $t$ períodos. Los restantes términos amortizativos son teóricos, pues han sido determinados con un tipo de interés que no corresponde a los mismos.

Tal y como está configurada la operación financiera, podemos determinar el saldo una vez finalizados los $t$ períodos, o sea, al principio de $(s+t)$ por el método prospectivo, mediante los términos amortizativos teóricos calculados en función del saldo en $s$ y el tipo de interés $i_{s+1}$ utilizado:

$$
C_{s+t}=\sum_{h=1}^{n-s-t} a_{s+t+h}^{(s)}\left(1+i_{s+1}\right)^{-h}
$$

Ahora bien, observando el gráfico siguiente, la ecuación [9] se puede descomponer, dando origen a la expresión [10]:

$$
C_{s+t}=\sum_{p=1}^{t} a_{s+t+p}^{(s)}\left(1+i_{s+1}\right)^{-p}+\sum_{q=t+1}^{n-s-t} a_{s+t+q}^{(s)}\left(1+i_{s+1}\right)^{-q}
$$

La ecuación precedente nos señala que el saldo al principio del período $(s+t)$ se obtiene como suma del valor actual de los $t$ primeros términos amortizativos teóricos con el valor actual de los restantes, calculados todos ellos en función del saldo en $s$.

En la siguiente revisión del tipo de interés, efectuada una vez transcurridos los $t$ períodos precedentes, nos situamos al principio del periodo $(s+t+1)$, debiendo aplicar la metodología descrita para calcular la cuantía de los nuevos términos amortizativos. Para ello establecemos la ecuación de equivalencia financiera al principio de dicho período entre el saldo y tales términos y suponiendo que el tipo de interés $i_{s+t+1}$ permanece constante para el resto de la operación:

$$
C_{s+t}=\sum_{h=1}^{n-s-t} a_{s+t+h}^{(s+t)}\left(1+i_{s+t+1}\right)^{-h}
$$

Dicho saldo se puede expresar de la siguiente manera:

$$
C_{s+t}=\sum_{p=1}^{t} a_{s+t+p}^{(s+t)}\left(1+i_{s+t+1}\right)^{-p}+\sum_{q=t+1}^{(n-s-t)} a_{s+t+p}^{(s+t)}\left(1+i_{s+t+1}\right)^{-q}
$$

Al igualar las dos expresiones obtenidas para $C_{s+t}$, ecuaciones [10] y [12], se llega a:

$$
\begin{aligned}
& \sum_{p=1}^{t} a_{s+t+p}^{(s)}\left(1+i_{s+1}\right)^{-p}+\sum_{q=t+1}^{n-s-t} a_{s+t+q}^{(s)}\left(1+i_{s+1}\right)^{-q} \\
& =\sum_{p=1}^{t} a_{s+t+p}^{(s+t)}\left(1+i_{s+t+1}\right)^{-p}+\sum_{q=t+1}^{n-s-t} a_{s+t+p}^{(s+t)}\left(1+i_{s+t+1}\right)^{-q}
\end{aligned}
$$


La ecuación [13] anterior nos da la relación entre los términos amortizativos teóricos $a_{s+t+p}^{(s)}$ calculados en función del saldo en $s, \mathrm{y}$ los términos efectivos $a_{s+t+p}^{(s+t)}$ calculados en función del saldo en $(s+t)$, todos ellos correspondientes al intervalo temporal $[s+t+1, s+2 t]$. Al establecer la ecuación de equivalencia financiera al principio de cada $t$ períodos para determinar las cuantías de los términos amortizativos teóricos, podemos considerar distintas leyes de variabilidad para los mismos (constantes, variables en progresión aritmética, variables en progresión geométrica). A continuación se desarrolla la modalidad de términos amortizativos teóricos constantes.

Situados al principio del período $(s+1)$, podemos establecer la ecuación de equivalencia financiera y obtener los $t$ términos amortizativos efectivos $a^{(s)}$ correspondientes al intervalo temporal $[s+1, s+t]$, la cual coincidirá con la cuantía de cada uno de los restantes términos amortizativos teóricos calculados en función del saldo en $s$. Al ser los términos amortizativos constantes, la ecuación de equivalencia financiera queda:

$$
C_{s}=a^{(s)} a \overline{n-s} \mid i_{s+1}
$$

Y además podemos determinar el saldo al principio del período $(s+t)$ por el método prospectivo, verificándose:

$$
C_{s+t}=a^{(s)} a \overline{n-(s+t)} \mid i_{s+1}
$$

De las ecuaciones [14] y [15] se llega a la siguiente ecuación que relaciona los saldos al inicio de dos plazos de revisión consecutivos:

$$
C_{s+t}=C_{s} \frac{a \overline{n-(s+t)} \mid i_{s+1}}{a \overline{n-s} \mid i_{s+1}}
$$

Por otra parte, una vez transcurridos $t$ períodos, y revisado el tipo de interés, se plantea la ecuación de equivalencia financiera para el cálculo de la cuantía de los términos amortizativos en función del saldo al principio del período $(s+t)$, obteniéndose:

$$
C_{s+t}=a^{(s+t)} a \overline{n-(s+t)} i_{s+t+1}
$$

Por tanto, tenemos dos expresiones que determinan el saldo al principio del período $(s+t)$ : la primera de ellas, ecuación [15], con términos amortizativos calculados en función del saldo en $s$, y la otra, ecuación [17], con los términos amortizativos calculados en función del saldo en $(s+t)$, por lo que finalmente al igualar queda:

$$
a^{(s)} a \overline{n-(s+t)} i_{s+1}=a^{(s+t)} a \overline{n-(s+t)} \mid i_{s+t+1}
$$

De dicha expresión obtenemos el término amortizativo efectivo en cada uno de los períodos del intervalo $[s+t+1, s+2 t]$ en función del término amortizativo efectivo en cada uno de los $t$ períodos precedentes:

$$
a_{s+t}=a^{(s)} \frac{a_{\overline{n-(s+t)} \mid i_{s+1}}}{a \overline{n-(s+t)} \mid i_{s+t+1}}
$$


La expresión anterior nos pone de manifiesto que para calcular el término amortizativo de un determinado intervalo temporal, no es necesario determinar previamente el saldo al principio del mismo y establecer la ecuación de equivalencia financiera, ya que la expresión anterior nos da la relación existente entre los $t$ términos amortizativos efectivos correspondientes a dos intervalos temporales consecutivos.

En la ecuación [19] podemos apreciar, como es obvio, la variación del término amortizativo en función de la del tipo de interés:

$$
\begin{aligned}
& \text { Si } i_{s+t+1}=i_{s+1} \Rightarrow a^{(s+1)}=a^{(s)} \\
& \text { Si } i_{s+t+1}>i_{s+1} \Rightarrow a^{(s+1)}>a^{(s)} \\
& \text { Si } i_{s+t+1}<i_{s+1} \Rightarrow a^{(s+1)}<a^{(s)}
\end{aligned}
$$

Una vez determinados los términos amortizativos y la relación existente entre los de dos intervalos temporales consecutivos de amplitud $t$, es preciso determinar las correspondientes cuotas de amortización y su relación.

La cuota de amortización correspondiente al período $(s+1)$, se determina según la conocida expresión utilizable en el método francés: $A_{s+1}=\frac{C_{s}}{s_{\overline{n-s} \mid i_{s+1}}}$. $\mathrm{Y}$ al sustituir $C_{s}$ por su valor, se llega a: $A_{s+1}=a^{(s)}\left(1+i_{s+1}\right)^{-(n-s)}$. Además, como las $t$ cuotas varían en progresión geométrica de razón $\left(1+i_{s+1}\right)$, tenemos para la última cuota de amortización del intervalo temporal $[s, s+t]$ la siguiente expresión:

$$
A_{s+t}=a^{(s)}\left(1+i_{s+1}\right)^{-(n-s-t+1)}
$$

Como al principio del intervalo $[s+t+1, s+2 t]$ se revisa el tipo de interés, obteniéndose, tal y como hemos señalado, la nueva cuantía de los términos amortizativos, es preciso deducir las expresiones de las cuotas de amortización de los $t$ períodos de ese nuevo intervalo y su relación con las del período precedente. Así, la primera cuota vale: $A_{s+t+1}=\frac{C_{s+t}}{s \frac{s+(s+t) \mid}{i_{s+t+1}}}$. Y teniendo en cuenta

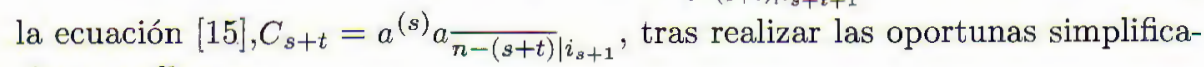
ciones se llega a:

$$
A_{s+t+1}=A_{s+t}\left(1+i_{s+1}\right) \frac{s \overline{n-(s+t)} \mid i_{s+1}}{s \overline{n-(s+t)} \mid i_{s+t+1}}
$$

La ecuación precedente nos señala la relación existente entre la última cuota de amortización del intervalo $[s, s+t]$ y la primera del siguiente, una vez el tipo de interés se ha modificado.

\subsubsection{Método con plan de amortización posdeterminado y duración indeterminada.}

En este método, al inicio de la respectiva operación, se desconoce la cuantía de las correspondientes cuotas de capital o plan de amortización, así como la duración de la operación, si bien necesariamente se ha de pactar una duración máxima, así como una cuantía mínima, bien para cada cuota de amortización o para cada término amortizativo . 
La característica fundamental de este método supone que al inicio de cada uno de los plazos de revisión, de amplitud $t$ períodos, y una vez conocido el tipo de interés que estará vigente durante el mismo, el deudor tiene la potestad de establecer bien la cuantía de cada una de las siguientes $t$ cuotas de amortización o la de cada uno de los siguientes $t$ términos amortizativos, por lo que estamos en presencia de una metodología que incorpora flexibilidad a las decisiones del cliente.

Si bien en el origen de la operación se desconoce la duración efectiva de la misma, habiéndose establecido, no obstante, una duración máxima, es evidente que la duración real va a estar vinculada a las sucesivas decisiones que el prestatario vaya tomando en relación con las cuantías de las cuotas de amortización o de los términos amortizativos. ${ }^{19}$

Es preciso señalar que en esta metodología, las decisiones tomadas sobre las cuantías de las cuotas de amortización o de las correspondientes a los términos amortizativos, son revisables cada $t$ períodos, en contraposición a lo analizado en las otras metodologías precedentes, en las que, tal y como se ha señalado, no existía tal posibilidad, pues las decisiones adoptadas en el inicio de la operación, permanecían vigentes durante toda la duración.

De acuerdo con lo expuesto, nos encontramos con dos modalidades que analizaremos a continuación: la de cuotas de amortización flexibles, y la de términos amortizativos flexibles.

a) Modalidad 1: Cuotas de amortización flexibles. Tal y como hemos señalado, al inicio de cada uno de los períodos de revisión del tipo de interés, el deudor indica las $t$ cuantías que en concepto de devolución del principal abonará hasta la próxima revisión del tipo de interés. Estas cuantías deben tener como importe mínimo aquel que permita que el préstamo quede amortizado en el plazo máximo pactado. No obstante, también las partes pueden acordar que en determinados períodos, cuyo número máximo ha de estar fijado en las condiciones del contrato, las cuotas de capital pactadas sean inferiores al mínimo señalado. En cualquier caso, y en función de las $t$ cuantías desembolsadas en concepto de cuotas de capital, en la próxima revisión, el importe mínimo se verá alterado de tal forma que se siga verificando la amortización del préstamo en el plazo máximo acordado.

Para la determinación de la cuantía mínima señalada anteriormente, hemos de tener en cuenta que la misma puede establecerse para cada uno de los $t$ periodos de tiempo hasta la próxima revisión, o bien señalarse para el conjunto de las t cuotas de amortización que deben abonarse hasta la siguiente revisión del tipo de interés. En ambos casos, tal como se ha señalado anteriormente, es preciso destacar que una vez transcurridos los citados $t$ períodos, la cuantía mínima es objeto de revisión.

En el primer supuesto, se establece una cuantía mínima constante para cada uno de los $t$ períodos de tiempo hasta la próxima revisión. Para determinar dicha cuantía, nos situamos al principio del período $(s+1)$ en el que el capital

19 Si se desea que el capital pendiente de amortizar no aumente de un período a otro, es preciso que la cuantía mínima pactada para el término amortizativo, cubra la cuota de interés. 
pendiente de devolver es de cuantía $C_{s}$ y faltando $(n-s)$ períodos hasta cumplir el plazo máximo de amortización, la cuota mínima de capital para cada uno de los $\mathrm{t}$ vencimientos correspondientes al intervalo $[s+1, s+t]$, ha de verificar la siguiente inecuación: $A_{s+h} \geq \frac{C_{s}}{n-s}, h=1,2, \cdots t$. En la hipótesis de establecer un mínimo para el conjunto de las $t$ cuotas de amortización, el mismo ha de ser de tal cuantía que se cumpla: $\sum_{h=1}^{t} A_{s+h} \geq \frac{t C_{s}}{n-s}$.

Una vez señalada la cuantía mínima que debe abonarse en concepto de cuota de devolución del saldo pendiente, es preciso detenerse en la forma de determinación de cada una de las t cuotas de amortización que ha de satisfacer el prestatario. En este sentido, podemos indicar que el mismo puede elegir de forma arbitraria tales cuantías, o bien aplicar alguno de los métodos señalados en el epígrafe 4.1.- Método con plan de amortización predeterminado, teniendo en cuenta que la duración que es preciso considerar ahora es el tiempo que resta hasta cumplir el plazo máximo de amortización pactado.

b) Modalidad 2: Términos amortizativos flexibles. En esta modalidad, y de acuerdo con lo expuesto, el prestatario al inicio de cada uno de los períodos de revisión del tipo de interés, señala el importe de cada uno de los $t$ siguientes términos amortizativos. Tales importes deben tener un mínimo que permita amortizar el préstamo en el plazo máximo señalado. Ahora bien, de igual forma a lo señalado en la Modalidad 1 de cuotas de amortización flexibles, los sujetos de la operación pueden pactar que en determinados períodos, cuyo número máximo se fija en el contrato de préstamo, los términos amortizativos sean inferiores al mínimo señalado.

Para la determinación de la cuantía mínima señalada anteriormente, es preciso suponer que el tipo de interés no se va a alterar hasta la finalización de la operación en el plazo máximo. Además, dicha cuantía mínima ha de fijarse para cada uno de los $t$ períodos de tiempo hasta la próxima revisión tanto del tipo de interés como de dicho mínimo.

Al principio del período $(s+1)$ y para cada uno de los $t$ vencimientos, la cuantía mínima constante ha de verificar la ecuación de equivalencia financiera entre el saldo vivo del préstamo en dicho momento y los $(n-s)$ términos restantes hasta el plazo máximo: $C_{s}=a a_{\overline{n-s}} \mid i_{s+1}$. De esta forma, si efectivamente el deudor abona las $t$ cuantías de importe mínimo, el saldo pendiente de amortizar transcurridos los $t$ períodos, se deduce de la ecuación: $C_{s+t}=$ $C_{s}\left(1+i_{s+1}\right)^{t}-a s_{\bar{t} \mid i_{s+1}}$.

Después de calculada la cuantía mínima del término amortizativo, se debe determinar el importe de cada uno de los t términos amortizativos que efectivamente abonará el deudor en el supuesto de que el mismo difiera del mínimo. En este sentido, el importe puede obtenerse de forma arbitraria o bien aplicar alguno de los métodos señalados en el epígrafe 3.- Metodologías de amortización con términos predeterminados, teniendo en cuenta que la duración a considerar es el tiempo restante hasta cumplir el plazo máximo de amortización pactado.

\section{Conclusiones}

En este trabajo se ha llevado a cabo un análisis financiero de las distintas metodologías de amortización aplicable a los préstamos con tipos de interés indiciados, distinguiendo entre metodologías de amortización con términos pre- 
determinados y posdeterminados.En las metodologías de amortización con términos predeterminados se conoce al inicio de la operación el importe de cada uno de los distintos términos amortizativos, desconociéndose las correspondientes cuotas de amortización, por lo que la duración es indeterminada. No obstante, se puede establecer un límite máximo. Una vez establecidas las cuantías de los términos se puede obtener una duración inicial de referencia, deducida de la ecuación de equivalencia financiera entre prestación y contraprestación al tipo de interés inicial. Por el contrario, también se puede pactar inicialmente la duración de referencia, y en función de la misma obtener el importe de los términos amortizativos.

En las metodologías de amortización con términos posdeterminados, el plan de amortización puede establecerse a priori o bien ser desconocido, distinguiéndose entre método con plan de amortización predeterminado y método con plan de amortización posdeterminado.

En el método con términos amortizativos posdeterminados y plan de amortización predeterminado, las cuotas de amortización pueden ser constantes o bien ser de cuantía variable, fijándose su importe de forma arbitraria o siguiendo un criterio de variación aritmético o uno geométrico. En ambos criterios, y con el fin de que todas las cuotas de amortización sean positivas, se ha de establecer limitaciones con respecto a la variabilidad de las mismas.

En el método con términos amortizativos posdeterminados y plan de amortización posdeterminado, sólo se conoce la cuantía del préstamo en el origen, pudiéndose establecer dos hipótesis en cuanto a la duración de la operación. En la hipótesis de duración determinada, una metodología de cálculo de los términos amortizativos consiste en la adaptación de los métodos clásicos de amortización a las características de los préstamos indiciados. En el supuesto de duración indeterminada, el deudor tiene la potestad de establecer, al principio de cada período de revisión, la cuantía de las $t$ siguientes cuotas de amortización o de los $t$ siguientes términos amortizativos, por lo que necesariamente ha de pactarse una duración máxima, así como un importe mínimo, ya sea de la cuota de amortización o del término amortizativo. Se distinguen, pues, dos modalidades: cuotas de amortización flexibles y términos amortizativos flexibles.

\section{Bibliografía}

Almoguera, A. (1995). La titulización crediticia; Ed. Civitas, S.A., Madrid.

Almoguera, A. (1996). Los préstamos a interés variable. Sistemática y modalidades; Perspectivas del Sistema Financiero, 54, pp. 31-50.

Ferruz, L., J. Sarto, y J. Marco (1995). Préstamos a interés variable: enfoque actual en el sistema financiero español, Actualidad Financiera, 3, pp. 37-64.

García, J y R. Cáceres (1998). Préstamos hipotecarios: análisis financiero; Actualidad financiera, 11, pp. 35-49.

García, J y R. Cáceres (2005). An Introduction to the Analysis and Complexities of Indexed Loans. VIII Italian-Spanish Meeting of Financial Mathematics.

Gil Peląez, L (1987). Matemática de las operaciones financieras, Ed. AC, Madrid.

Lamothe, P.(1987). Los créditos a tipo de interés variable; Actualidad Financiera, 1, enero.

Meneu, V., M. Jordá; y M. Barreira (1994). Operaciones financieras en el mercado español; Ed. Ariel, S.A., Barcelona.

Terceño, A. et al. (1997) Matemática Financiera; Ed. Pirámide, S.A., Madrid. 